\title{
WEAVING FRAMES
}

\author{
Travis Bemrose, Peter G. Casazza, Karlheinz Gröchenig, \\ MARK C. LAMMERS, RICHARD G. LYNCH
}

Abstract. We study an intriguing question in frame theory we call Weaving Frames that is partially motivated by preprocessing of Gabor frames. Two frames $\left\{\varphi_{i}\right\}_{i \in I}$ and $\left\{\psi_{i}\right\}_{i \in I}$ for a Hilbert space $\mathbb{H}$ are woven if there are constants $0<A \leqslant B$ so that for every subset $\sigma \subset I$, the family $\left\{\varphi_{i}\right\}_{i \in \sigma} \cup\left\{\psi_{i}\right\}_{i \in \sigma^{c}}$ is a frame for $\mathbb{H}$ with frame bounds $A, B$. Fundamental properties of woven frames are developed and key differences between weaving Riesz bases and weaving frames are considered. In particular, it is shown that a Riesz basis cannot be woven with a redundant frame. We also introduce an apparently weaker form of weaving but show that it is equivalent to weaving. Weaving frames has potential applications in wireless sensor networks that require distributed processing under different frames, as well as preprocessing of signals using Gabor frames.

Mathematics subject classification (2010): 42C15.

Keywords and phrases: Frame, Riesz basis, distance between subspaces.

\section{REFERENCES}

[1] P. G. CASAZZA AND G. KutynioK, Finite frames: Theory and applications, Springer (2013).

[2] O. Christensen, An Introduction to Frames and Riesz Bases, Birkhäuser, Boston (2003).

[3] F. Deutsch, The angle between subspaces of a Hilbert space, S. P. Singh (Ed.), Approximation Theory, Wavelets and Applications, Kluwer Academic Publ., Dordrecht (1995), pp. 107-130

[4] M. DÖRFLER, Quilted Gabor frames - a new concept for adaptive time-frequency representation, Adv. Appl. Math 47 no. 4 (2011) 668-687.

[5] M. Dörfler, J. L. Romero, Frames adapted to a phase-space cover, Constr. Approx. 39 no. 3 (2014) 445-484.

[6] R. J. Duffin And A. C. Schaeffer, A class of nonharmonic Fourier series, Trans. Amer. Math. Soc., 72 (1952) 341-366.

[7] K. GRÖCHENIG, Foundations of Time-Frequency Analysis, Birkhaüser, Boston (2001).

[8] J. L. Romero, Surgery of spline-type and molecular frames, J. Fourier Anal. and Appl. 17 no. 1 (2011) 135-174. 